Eur. J. Math. Appl. (2021)1:11

URL: http://ejma.euap.org

(C) 2021 European Journal of Mathematics and Applications

\title{
SOLVABILITY FOR A NONLINEAR DIFFERENTIAL PROBLEM OF LANGEVIN TYPE VIA PHI-CAPUTO APPROACH
}

\author{
HAMID BEDDANI ${ }^{1}$ AND ZOUBIR DAHMANI ${ }^{2, *}$
}

\begin{abstract}
This paper deals with a new problem of Langevin type. A first existence and uniqueness result via $\varphi$-Caputo derivative is studied. Then, an existence of one solution is investigated. Some illustrative examples are discussed at the end.
\end{abstract}

\section{INTRODUCTION}

The fractional calculus has a significant role in various scientific fields, see for instance [10, 16,17]. As applied results of this significant role, we shed light on the fact that fractional order differential equations have attired attention of many researchers that have worked in different fields of research $[7,8,15]$. However, most of these research works have been achieved by means of fractional derivatives of type: RiemannLiouville, Hadamard, Katugampola, Grunwald Letnikov and Caputo. But, fractional derivative of a function with respect to another function [12] is different from the others since its kernel appears in terms of another function $\varphi$. Recently, some fractional differential results have been considered in [2-4,11].

In most of the present articles, Schauder's, Krasnoselskii's, Darbo's, or Mönch's theories have been used to prove existence of solutions of nonlinear fractional differential equations with some restrictive conditions $[1,5,6,14]$.

To cite a some research papers that have motivate our present work, we begin by recalling the research paper [9] where the authors investigated the existence and uniqueness of solutions for nonlinear Langevin equation of fractional orders by considering anti-periodic boundary conditions:

$$
\left\{\begin{array}{c}
{ }^{c} D_{0^{+}}^{\beta}\left({ }^{c} D_{0^{+}}^{\alpha}+\mu\right) u(t)=g(t, u(t)), t \in(0,1), 0<\alpha, \beta \leq 1,1<\beta \leq 2 \\
u(0)+u(1)=0, \\
{ }^{c} D_{0^{+}}^{\alpha} u(0)+{ }^{c} D_{0^{+}}^{\alpha} u(1)=0, \\
{ }^{c} D_{0^{+}}^{2 \alpha} u(0)+{ }^{c} D_{0^{+}}^{2 \alpha} u(1)=0,
\end{array}\right.
$$

\footnotetext{
${ }^{1}$ Laboratory of Complex Systems of the Higher School of Electrical and Energy Engineering of Oran, 31000, Oran, Algeria

${ }^{2}$ Laboratory of Pure and Applied Mathematics, Abdelhamid Bni Badis University, 27000, ALGERIA

*Corresponding AUTHOR

E-mail addresses: beddanihamid@gmail.com, zzdahmani@yahoo.fr.

Key words and phrases. Langevin equation; $\varphi$-Caputo derivative; existence of solution; fixed point.

Received 23/08/2021.
} 
where the function $g:[0,1] \times \mathbb{R} \times \mathbb{R} \rightarrow \mathbb{R}$ is a continuous and $\mu$ is a real number.and ${ }^{c} D_{0^{+}}^{2 \alpha}$ is the sequential fractional derivative.

S. Kosari et al. [13] worked on the existence and uniqueness of solutions on the following generalization of Langevin equation:

$$
\left\{\begin{array}{c}
{ }^{c} D_{0^{+}}^{\beta}\left({ }^{c} D_{0^{+}}^{\alpha}+\mu\right) u(t)=g\left(t, u(t), u^{\prime}(t)\right), t \in(0,1), \\
0<\alpha, \beta \leq 1,2<\beta \leq 3, \\
u(0)=u(1)=u^{\prime}(0)=u^{\prime}(1)=0,
\end{array}\right.
$$

where ${ }^{c} D^{\alpha}$ is the Caputo fractional derivative of order $\alpha, g:[0,1] \times \mathbb{R} \times \mathbb{R} \rightarrow \mathbb{R}$ is a continuous function and $\mu$ is a real number.

Then, A. Seemab with his coauthors [18] investigated the Langevin equation that involves a $\varphi$-Caputo fractional operator as:

$$
\left\{\begin{array}{c}
{ }^{c} D_{a^{+}, t}^{\beta ; \varphi}\left({ }^{c} D_{a^{+}, t}^{\alpha ; \varphi}+\mu\right)[u]=g\left(t, u(t),{ }^{c} D_{a^{+}, t}^{\delta ; \varphi}[u]\right), t \in(a, T), \\
u(a)=0, u(\eta)=0 \\
u(T)=\lambda\left(I_{a^{+}, \zeta}^{\delta ; \varphi}\right)[u], \quad \mu, \lambda>0
\end{array}\right.
$$

such that $\left(I_{a^{+}, \zeta}^{\delta ; \varphi}\right)$ and $\left({ }^{c} D_{a^{+}, t}^{\theta ; \varphi}\right)$, are the $\varphi$-Caputo fractional integral of order $\delta, \varphi$-Caputo fractional derivative of orders $\theta \in\{\alpha, \beta, \delta\}$ respectively, $0 \leq a<\eta<\zeta<T<\infty$ and $g:[a, T] \times \mathbb{R} \times \mathbb{R} \rightarrow \mathbb{R}^{+}$is a continuous function.

In the present research work, we study the existence and uniqueness of solutions for an new proposed generalization of Langevin equation which includes the $\varphi$-Caputo fractional-order of the form:

$$
\left\{\begin{array}{c}
{ }^{c} D_{a^{+}}^{\alpha_{1} ; \varphi}\left({ }^{c} D_{a^{+}}^{\alpha_{2} ; \varphi}\left({ }^{c} D_{a^{+}}^{\alpha_{3} ; \varphi}+\mu\right)\right) u(t)=g\left(t, u(t),{ }^{c} D_{a^{+}}^{\alpha_{4} ; \varphi} u(t)\right), t \in J=(a, b) \\
u(a)=\left({ }^{c} D_{a^{+}}^{\alpha_{4} ; \varphi}\right) u(b)=0, \quad u(b)=\rho \sum_{i=1}^{n} u\left(\zeta_{i}\right), \\
\mu, \rho_{i}>0, \quad 0 \leq a<\zeta_{i}<b<\infty, \text { and } \varphi(b)-\varphi(a)=K>0
\end{array}\right.
$$

Here, we take ${ }^{c} D_{a^{+}}^{\alpha_{i} ; \varphi}, i=\overline{1,4}$ are the $\varphi$-Caputo fractional derivative of orders $\alpha_{i}, 0<\alpha_{i}<1$, $\alpha_{4}<\alpha_{3}$, and $\mu, \rho \in \mathbb{R}_{+}^{*}$, and $\varphi: J \rightarrow \mathbb{R}$ be an increasing function such that $\varphi^{\prime}(t) \neq 0$, for all $t \in J$, to be defined later, $g: J \times \mathbb{R} \times \mathbb{R} \rightarrow \mathbb{R}^{+}$is a given function satisfying some assumptions that will be specified later,

\section{Preliminaries}

In this section, we need to recall some definitions and lemmas which are very needed for our results. Let $\varphi: J \rightarrow \mathbb{R}$ be an increasing function with $\varphi^{\prime}(t) \neq 0$, for all $t \in J$, and let $C(J, \mathbb{R})$ be the Banach space

Definition 1. ([4]). For $\alpha>0$, the left-sided $\varphi$-Riemann Liouville fractional integral of order $\alpha$ for an integrable function $u: J \rightarrow \mathbb{R}$ with respect to another function $\varphi: J \rightarrow \mathbb{R}$ that is an increasing differentiable function such that $\varphi^{\prime}(t) \neq 0$, for all $t \in J$ is defined as follows

$$
I_{a^{+}}^{\alpha ; \varphi} u(t)=\frac{1}{\Gamma(\alpha)} \int_{a}^{t} \varphi^{\prime}(s)(\varphi(t)-\varphi(s))^{\alpha-1} u(s) d s
$$


where $\Gamma$ is the gamma function. Note that equation (2.1) is reduced to the Riemann Liouville and Hadamard fractional integrals when $\varphi(t)=t$ and $\varphi(t)=\ln t$, respectively.

Definition 2. ([4]). Let $n \in \mathbb{N}$ and let $\varphi, u \in C^{n}(J)$ be two functions such that $\varphi$ is increasing and $\varphi^{\prime}(t) \neq 0$, for all $t \in J$. The left-sided $\varphi$-Riemann Liouville fractional derivative of a function $u$ of order $\alpha$ is defined by

$$
\begin{aligned}
D_{a^{+}}^{\alpha ; \varphi} u(t) & =\left(\frac{1}{\varphi^{\prime}(t)} \frac{d}{d t}\right)^{n} I_{a^{+}}^{n-\alpha ; \varphi} u(t) \\
& =\frac{1}{\Gamma(n-\alpha)}\left(\frac{1}{\varphi^{\prime}(t)} \frac{d}{d t}\right)^{n} \int_{a}^{t} \varphi^{\prime}(s)(\varphi(t)-\varphi(s))^{n-\alpha-1} u(s) d s
\end{aligned}
$$

where $n=[\alpha]+1$.

Definition 3. ([4]). Let $n \in \mathbb{N}$ and let $\varphi, u \in C^{n}(J)$ be two functions such that $\varphi$ is increasing and $\varphi^{\prime}(t) \neq 0$, for all $t \in J$. The left-sided $\varphi$-Caputo fractional derivative of a function $x$ of order $\alpha$ is defined by

$$
{ }^{c} D_{a^{+}}^{\alpha ; \varphi} u(t)=I_{a^{+}}^{n-\alpha ; \varphi}\left(\frac{1}{\varphi^{\prime}(t)} \frac{d}{d t}\right)^{n} u(t),
$$

where $n=[\alpha]+1$ for $\alpha \notin \mathbb{N}, n=\alpha$ for $\alpha \in \mathbb{N}$.

To simplify notation, we will use the abbreviated symbol

$$
u_{\varphi}^{[n]}(t)=\left(\frac{1}{\varphi^{\prime}(t)} \frac{d}{d t}\right)^{n} u(t) .
$$

From the definition, it is clear that,

$$
{ }^{c} D_{a^{+}}^{\alpha ; \varphi} u(t)= \begin{cases}\frac{1}{\Gamma(n-\alpha)} \int_{a}^{t} \varphi^{\prime}(s)(\varphi(t)-\varphi(s))^{n-\alpha-1} u_{\varphi}^{[n]}(s) d s, & \text { if } \alpha \notin \mathbb{N}, \\ u_{\varphi}^{[n]}(t) & \text { if } \alpha \in \mathbb{N} .\end{cases}
$$

This generalization (2.2) yields the Caputo fractional derivative operator when $\varphi(t)=t$. Moreover, for $\varphi(t)=\ln t$, it gives the Caputo Hadamard fractional derivative.

Lemma 1. ( [5]). Let $\alpha, \beta>0$, and $u \in L^{1}(J)$. Then

$$
I_{a^{+}}^{\alpha ; \varphi} I_{a^{+}}^{\beta ; \varphi} u(t)=I_{a^{+}}^{\alpha+\beta ; \varphi} u(t), \quad \text { a.e. } t \in J .
$$

In particular,

If $u \in C(J)$. Then $I_{a^{+}}^{\alpha ; \varphi} I_{a^{+}}^{\beta ; \varphi} u(t)=I_{a^{+}}^{\alpha+\beta ; \varphi} u(t), t \in J$.

Next, we recall the property describing the composition rules for fractional $\varphi$-integrals and $\varphi$ derivatives.

Lemma 2. ( [5]). Let $\alpha>0$ The following holds:

If $u \in C([a, b])$ then

$$
{ }^{c} D_{a^{+}}^{\alpha ; \varphi} I_{a^{+}}^{\alpha ; \varphi} u(t)=u(t), t \in[a, b] .
$$

If $u \in C^{n}(J), n-1<\alpha<n$. Then

$$
I_{a^{+}}^{\alpha ; \varphi c} D_{a^{+}}^{\alpha ; \varphi} u(t)=u(t)-\sum_{k=0}^{n-1} \frac{u_{\varphi}^{[k]}(a)}{k !}[\varphi(t)-\varphi(a)]^{k},
$$

for all $t \in[a, b]$. In particular, if $0<\alpha<1$, we have

$$
I_{a^{+}}^{\alpha ; \varphi c} D_{a^{+}}^{\alpha ; \varphi} u(t)=u(t)-u(a) .
$$


Lemma 3. ( $[5,12])$. Let $t>a, \alpha \geq 0$; and $\beta>0$. Then

- $I_{a^{+}}^{\alpha ; \varphi}[\varphi(t)-\varphi(a)]^{\beta-1}=\frac{\Gamma(\beta)}{\Gamma(\beta+\alpha)}[\varphi(t)-\varphi(a)]^{\beta+\alpha-1}$,

- ${ }^{c} D_{a^{+}}^{\alpha ; \varphi}[\varphi(t)-\varphi(a)]^{\beta-1}=\frac{\Gamma(\beta)}{\Gamma(\beta-\alpha)}[\varphi(t)-\varphi(a)]^{\beta-\alpha-1}$,

- ${ }^{c} D_{a^{+}}^{\alpha ; \varphi}[\varphi(t)-\varphi(a)]^{k}=0$, for all $k \in\{0, \ldots, n-1\}, n \in \mathbb{N}$.

Lemma 4. ([12,15]). Let $\alpha>0, n \in \mathbb{N}$; such that $n-1<q \leq n$. Then:

- ${ }^{c} D_{a^{+}}^{q ; \varphi} I_{a^{+}}^{\alpha ; \varphi} u(t)={ }^{c} D_{a^{+}}^{q-\alpha ; \varphi} u(t)$; if $q>\alpha$.

- ${ }^{c} D_{a^{+}}^{q ; \varphi} I_{a^{+}}^{\alpha ; \varphi} u(t)=I_{a^{+}}^{\alpha-q ; \varphi} u(t)$; if $\alpha>q$.

Lemma 5. ( [18]) Given a function $u \in C^{n}[a, b]$ and $0<q<1$, we have

$$
\left|I_{a^{+}}^{q ; \varphi} u\left(t_{2}\right)-I_{a^{+}}^{q ; \varphi} u\left(t_{1}\right)\right| \leq \frac{2\|u\|}{\Gamma(q+1)}\left(\varphi\left(t_{2}\right)-\varphi\left(t_{1}\right)\right)^{q} .
$$

Lemma 6. For a given $g \in L^{1}(J, \mathbb{R}, \mathbb{R})$, the unique solution of the linear fractional initial value problem

$$
\left\{\begin{array}{c}
{ }^{c} D_{a^{+}}^{\alpha_{1} ; \varphi}\left({ }^{c} D_{a^{+}}^{\alpha_{2} ; \varphi}\left({ }^{c} D_{a^{+}}^{\alpha_{3} ; \varphi}+\mu\right)\right) u(t)=g(t), t \in J=(a, b) \\
u(a)=\left({ }^{c} D_{a^{+}}^{\alpha_{4} ; \varphi}\right) u(b)=0, \quad u(b)=\rho \sum_{i=1}^{n} u\left(\zeta_{i}\right), \quad \mu, \rho_{i}>0, \quad 0 \leq a<\zeta_{i}<b<\infty, \\
\varphi(b)-\varphi(a)=K>0
\end{array}\right.
$$

is given by

$$
\begin{aligned}
u(t)= & I_{a^{+}}^{\alpha_{1}+\alpha_{2}+\alpha_{3} ; \varphi} g(t)-\mu I_{a^{+}}^{\alpha_{3} ; \varphi} u(t) \\
& +\frac{\rho(\varphi(t)-\varphi(a))^{\alpha_{2}+\alpha_{3}} \sum_{i=1}^{n} u\left(\zeta_{i}\right)}{\Delta \Gamma\left(\alpha_{2}+\alpha_{3}+1\right) \Gamma\left(\alpha_{3}-\alpha_{4}+1\right)}-\frac{\rho K^{\alpha_{2}}(\varphi(t)-\varphi(a))^{\alpha_{3}} \sum_{i=1}^{n} u\left(\zeta_{i}\right)}{\Delta \Gamma\left(\alpha_{3}+1\right) \Gamma\left(\alpha_{2}+\alpha_{3}-\alpha_{4}+1\right)} \\
& +\frac{\mu K^{\alpha_{4}}\left(K^{\alpha_{2}}(\varphi(t)-\varphi(a))^{\alpha_{3}}-(\varphi(t)-\varphi(a))^{\alpha_{2}+\alpha_{3}}\right) I_{a^{+}}^{\alpha_{3}-\alpha_{4} ; \varphi} u(b)}{\Delta \Gamma\left(\alpha_{3}+1\right) \Gamma\left(\alpha_{2}+\alpha_{3}+1\right)} \\
& +\frac{\mu(\varphi(t)-\varphi(a))^{\alpha_{2}+\alpha_{3}} I_{a^{+}}^{\alpha_{3} ; \varphi} u(b)}{\Delta \Gamma\left(\alpha_{2}+\alpha_{3}+1\right) \Gamma\left(\alpha_{3}-\alpha_{4}+1\right)}-\frac{\mu K^{\alpha_{2}}(\varphi(t)-\varphi(a))^{\alpha_{3}} I_{a^{+}}^{\alpha_{3} ; \varphi} u(b)}{\Delta \Gamma\left(\alpha_{3}+1\right) \Gamma\left(\alpha_{2}+\alpha_{3}-\alpha_{4}+1\right)} \\
& +\frac{K^{\alpha_{4}}\left((\varphi(t)-\varphi(a))^{\alpha_{2}+\alpha_{3}}-K^{\alpha_{2}}(\varphi(t)-\varphi(a))^{\alpha_{3}}\right) I_{a^{+}}^{\alpha_{1}+\alpha_{2}+\alpha_{3}-\alpha_{4} ; \varphi} g(b)}{\Delta \Gamma\left(\alpha_{3}+1\right) \Gamma\left(\alpha_{2}+\alpha_{3}+1\right)} \\
& +\frac{K^{\alpha_{2}}(\varphi(t)-\varphi(a))^{\alpha_{3}} I_{a^{+}}^{\alpha_{1}+\alpha_{2}+\alpha_{3} ; \varphi} g(b)}{\Delta \Gamma\left(\alpha_{3}+1\right) \Gamma\left(\alpha_{2}+\alpha_{3}-\alpha_{4}+1\right)} \\
& -\frac{(\varphi(t)-\varphi(a))^{\alpha_{2}+\alpha_{3}} I_{a^{+}}^{\alpha_{1}+\alpha_{2}+\alpha_{3} ; \varphi} g(b)}{\Delta \Gamma\left(\alpha_{2}+\alpha_{3}+1\right) \Gamma\left(\alpha_{3}-\alpha_{4}+1\right)}
\end{aligned}
$$

where

$$
\Delta=\left(\frac{K^{\alpha_{2}+\alpha_{3}}}{\Gamma\left(\alpha_{2}+\alpha_{3}+1\right) \Gamma\left(\alpha_{3}-\alpha_{4}+1\right)}-\frac{K^{\alpha_{2}+\alpha_{3}}}{\Gamma\left(\alpha_{3}+1\right) \Gamma\left(\alpha_{2}+\alpha_{3}-\alpha_{4}+1\right)}\right) .
$$

Proof. For $0<\alpha_{i}<1, i=\overline{1,3}$, Lemma 2 yields

$$
\left({ }^{c} D_{a^{+}}^{\alpha_{2} ; \varphi}\left({ }^{c} D_{a^{+}}^{\alpha_{3} ; \varphi}+\mu\right)\right) u(t)=I_{a^{+}}^{\alpha_{1} ; \varphi} g(t)+c_{1}
$$

and

$$
\left({ }^{c} D_{a^{+}}^{\alpha_{3} ; \varphi}+\mu\right) u(t)=I_{a^{+}}^{\alpha_{1}+\alpha_{2} ; \varphi} g(t)+I_{a^{+}}^{\alpha_{2} ; \varphi} c_{1}+c_{2},
$$


SO

$$
u(t)=I_{a^{+}}^{\alpha_{1}+\alpha_{2}+\alpha_{3} ; \varphi} g(t)-\mu I_{a^{+}}^{\alpha_{3} ; \varphi} u(t)+I_{a^{+}}^{\alpha_{2}+\alpha_{3} ; \varphi} c_{1}+I_{a^{+}}^{\alpha_{3} ; \varphi} c_{2}+c_{3},
$$

where $c_{1}, c_{2}, c_{3} \in \mathbb{R}$, by conditions $u(a)=0$, we gat

$$
u(t)=I_{a^{+}}^{\alpha_{1}+\alpha_{2}+\alpha_{3} ; \varphi} g(t)-\mu I_{a^{+}}^{\alpha_{3} ; \varphi} u(t)+I_{a^{+}}^{\alpha_{2}+\alpha_{3} ; \varphi} c_{1}+I_{a^{+}}^{\alpha_{3} ; \varphi} c_{2},
$$

by integrating we find

$$
\begin{aligned}
u(t)= & I_{a^{+}}^{\alpha_{1}+\alpha_{2}+\alpha_{3} ; \varphi} g(t)-\mu I_{a^{+}}^{\alpha_{3} ; \varphi} u(t) \\
& +c_{1} \frac{(\varphi(t)-\varphi(a))^{\alpha_{2}+\alpha_{3}}}{\Gamma\left(\alpha_{2}+\alpha_{3}+1\right)}+c_{2} \frac{(\varphi(t)-\varphi(a))^{\alpha_{3}}}{\Gamma\left(\alpha_{3}+1\right)} .
\end{aligned}
$$

For $0<\alpha_{4}<1$, and by (2.4), and Lemma 4 we have

$$
{ }^{c} D_{a^{+}}^{\alpha_{4} ; \varphi} u(t)=I_{a^{+}}^{\alpha_{1}+\alpha_{2}+\alpha_{3}-\alpha_{4} ; \varphi} g(t)-\mu I_{a^{+}}^{\alpha_{3}-\alpha_{4} ; \varphi} u(t)+I_{a^{+}}^{\alpha_{2}+\alpha_{3}-\alpha_{4} ; \varphi} c_{1}+I_{a^{+}}^{\alpha_{3}-\alpha_{4} ; \varphi} c_{2},
$$

so

$$
\begin{aligned}
{ }^{c} D_{a^{+}}^{\alpha_{4} ; \varphi} u(t)= & I_{a^{+}}^{\alpha_{1}+\alpha_{2}+\alpha_{3}-\alpha_{4} ; \varphi} g(t)-\mu I_{a^{+}}^{\alpha_{3}-\alpha_{4} ; \varphi} u(t) \\
& +c_{1} \frac{(\varphi(t)-\varphi(a))^{\alpha_{2}+\alpha_{3}-\alpha_{4}}}{\Gamma\left(\alpha_{2}+\alpha_{3}-\alpha_{4}+1\right)}+c_{2} \frac{(\varphi(t)-\varphi(a))^{\alpha_{3}-\alpha_{4}}}{\Gamma\left(\alpha_{3}-\alpha_{4}+1\right)}
\end{aligned}
$$

by conditions $\varphi(b)-\varphi(a)=K,\left({ }^{c} D_{a^{+}}^{\alpha_{4} ; \varphi}\right) u(b)=0$, and $u(b)=\rho \sum_{i=1}^{n} u\left(\zeta_{i}\right)$, we give

$$
c_{1} \frac{K^{\alpha_{2}+\alpha_{3}}}{\Gamma\left(\alpha_{2}+\alpha_{3}+1\right)}+c_{2} \frac{K^{\alpha_{3}}}{\Gamma\left(\alpha_{3}+1\right)}=u(b)-I_{a^{+}}^{\alpha_{1}+\alpha_{2}+\alpha_{3} ; \varphi} g(b)+\mu I_{a^{+}}^{\alpha_{3} ; \varphi} u(b)
$$

and

$$
c_{1} \frac{K^{\alpha_{2}+\alpha_{3}-\alpha_{4}}}{\Gamma\left(\alpha_{2}+\alpha_{3}-\alpha_{4}+1\right)}+c_{2} \frac{K^{\alpha_{3}-\alpha_{4}}}{\Gamma\left(\alpha_{3}-\alpha_{4}+1\right)}=\mu I_{a^{+}}^{\alpha_{3}-\alpha_{4} ; \varphi} u(b)-I_{a^{+}}^{\alpha_{1}+\alpha_{2}+\alpha_{3}-\alpha_{4} ; \varphi} g(b)
$$

so

$$
\begin{aligned}
c_{1}= & \frac{u(b)}{\Delta \Gamma\left(\alpha_{3}-\alpha_{4}+1\right)} \\
& +\frac{\mu}{\Delta \Gamma\left(\alpha_{3}-\alpha_{4}+1\right)} I_{a^{+}}^{\alpha_{3} ; \varphi} u(b)-\frac{K^{\alpha_{4}}}{\Delta \Gamma\left(\alpha_{3}+1\right)} \mu I_{a^{+}}^{\alpha_{3}-\alpha_{4} ; \varphi} u(b) \\
& +\frac{K^{\alpha_{4}}}{\Delta \Gamma\left(\alpha_{3}+1\right)} I_{a^{+}}^{\alpha_{1}+\alpha_{2}+\alpha_{3}-\alpha_{4} ; \varphi} g(b)-\frac{1}{\Delta \Gamma\left(\alpha_{3}-\alpha_{4}+1\right)} I_{a^{+}}^{\alpha_{1}+\alpha_{2}+\alpha_{3} ; \varphi} g(b)
\end{aligned}
$$

and

$$
\begin{aligned}
c_{2}= & -\frac{u(b) K^{\alpha_{2}}}{\Delta \Gamma\left(\alpha_{2}+\alpha_{3}-\alpha_{4}+1\right)} \\
& \frac{K^{\alpha_{2}+\alpha_{4}}}{\Delta \Gamma\left(\alpha_{2}+\alpha_{3}+1\right)} \mu I_{a^{+}}^{\alpha_{3}-\alpha_{4} ; \varphi} u(b)-\frac{\mu K^{\alpha_{2}}}{\Delta \Gamma\left(\alpha_{2}+\alpha_{3}-\alpha_{4}+1\right)} I_{a^{+}}^{\alpha_{3} ; \varphi} u(b) \\
& +\frac{K^{\alpha_{2}}}{\Delta \Gamma\left(\alpha_{2}+\alpha_{3}-\alpha_{4}+1\right)} I_{a^{+}}^{\alpha_{1}+\alpha_{2}+\alpha_{3} ; \varphi} g(b)-\frac{K^{\alpha_{2}+\alpha_{4}}}{\Delta \Gamma\left(\alpha_{2}+\alpha_{3}+1\right)} I_{a^{+}}^{\alpha_{1}+\alpha_{2}+\alpha_{3}-\alpha_{4} ; \varphi} g(b)
\end{aligned}
$$

Substituting the values of $c_{1}, c_{2}$ into (2.5), we find the solution.

Conclusion 1. By (2.6) we obtain

$$
\begin{aligned}
& { }^{c} D_{a^{+}}^{\alpha_{4} ; \varphi} u(t) \\
= & I_{a^{+}}^{\alpha_{1}+\alpha_{2}+\alpha_{3}-\alpha_{4} ; \varphi} g(t)-\mu I_{a^{+}}^{\alpha_{3}-\alpha_{4} ; \varphi} u(t)
\end{aligned}
$$




$$
\begin{aligned}
& +\frac{u(b)\left((\varphi(t)-\varphi(a))^{\alpha_{2}+\alpha_{3}-\alpha_{4}}-K^{\alpha_{2}}(\varphi(t)-\varphi(a))^{\alpha_{3}-\alpha_{4}}\right)}{\Delta \Gamma\left(\alpha_{3}-\alpha_{4}+1\right) \Gamma\left(\alpha_{2}+\alpha_{3}-\alpha_{4}+1\right)} \\
& +\frac{\mu\left((\varphi(t)-\varphi(a))^{\alpha_{2}+\alpha_{3}-\alpha_{4}}-K^{\alpha_{2}}(\varphi(t)-\varphi(a))^{\alpha_{3}-\alpha_{4}}\right) I_{a^{+}}^{\alpha_{3} ; \varphi} u(b)}{\Delta \Gamma\left(\alpha_{3}-\alpha_{4}+1\right) \Gamma\left(\alpha_{2}+\alpha_{3}-\alpha_{4}+1\right)} \\
& +\frac{\mu K^{\alpha_{2}+\alpha_{4}}(\varphi(t)-\varphi(a))^{\alpha_{3}-\alpha_{4}} I_{a^{+}}^{\alpha_{3}-\alpha_{4} ; \varphi} u(b)}{\Delta \Gamma\left(\alpha_{3}-\alpha_{4}+1\right) \Gamma\left(\alpha_{2}+\alpha_{3}+1\right)} \\
& -\frac{\mu K^{\alpha_{4}}(\varphi(t)-\varphi(a))^{\alpha_{2}+\alpha_{3}-\alpha_{4}} I_{a^{+}}^{\alpha_{3}-\alpha_{4} ; \varphi} u(b)}{\Delta \Gamma\left(\alpha_{3}+1\right) \Gamma\left(\alpha_{2}+\alpha_{3}-\alpha_{4}+1\right)} \\
& +\frac{\left(K^{\alpha_{2}}(\varphi(t)-\varphi(a))^{\alpha_{3}-\alpha_{4}}-(\varphi(t)-\varphi(a))^{\alpha_{2}+\alpha_{3}-\alpha_{4}}\right) I_{a^{+}+\alpha_{2}+\alpha_{3} ; \varphi}^{\alpha_{1}} g(b)}{\Delta \Gamma\left(\alpha_{3}-\alpha_{4}+1\right) \Gamma\left(\alpha_{2}+\alpha_{3}-\alpha_{4}+1\right)} \\
& +\frac{K^{\alpha_{4}}(\varphi(t)-\varphi(a))^{\alpha_{2}+\alpha_{3}-\alpha_{4}} I_{a^{+}}^{\alpha_{1}+\alpha_{2}+\alpha_{3}-\alpha_{4} ; \varphi} g(b)}{\Delta \Gamma\left(\alpha_{3}+1\right) \Gamma\left(\alpha_{2}+\alpha_{3}-\alpha_{4}+1\right)} \\
& -\frac{K^{\alpha_{2}+\alpha_{4}}(\varphi(t)-\varphi(a))^{\alpha_{3}-\alpha_{4}} I_{a^{+}}^{\alpha_{1}+\alpha_{2}+\alpha_{3}-\alpha_{4} ; \varphi} g(b)}{\Delta \Gamma\left(\alpha_{3}-\alpha_{4}+1\right) \Gamma\left(\alpha_{2}+\alpha_{3}+1\right)} .
\end{aligned}
$$

\section{MAin Results}

In this section, we propose the two existence and uniqueness/existence results of Langevin type problem (1.1). Let the Banach space $E=\left\{u: u \in C^{1}[a, b],{ }^{c} D_{a^{+}}^{\alpha ; \varphi} u \in C[a, b]\right\}$ be equipped with the norm:

$$
\|u\|=\max _{t \in[a, b]}|u(t)|+\max _{t \in[a, b]}\left|{ }^{c} D_{a^{+}}^{\alpha_{4} ; \varphi} u(t)\right| .
$$

To prove the main results, we need the following assumptions:

- $\left.\mathbb{H}_{1}\right) g:[a, b] \times \mathbb{R} \times \mathbb{R} \rightarrow \mathbb{R}$ is continuous function.

- $\mathbb{H}_{2}$ ) There exists a constant $\delta>0$ such that

$$
|g(t, u, v)-g(t, x, y)| \leq \delta(|u-x|+|v-y|),
$$

for all $t \in[a, b], u, v, x, y \in \mathbb{R}$.

- $\left.\mathbb{H}_{3}\right)$ There exists a nonnegative function $\phi \in L[0,1]$ such that

$$
|g(t, u, v)| \leq \phi(t)+\rho_{1}|u|^{\delta_{1}}+\rho_{2}|v|^{\delta_{2}},
$$

where $\rho_{1}, \rho_{2} \in \mathbb{R}$, and $0<\delta_{1}, \delta_{2}<1$

For the sake of convenience, we define the following constants:

$$
\begin{aligned}
\Upsilon_{1}= & \frac{K^{\alpha_{1}+\alpha_{2}+\alpha_{3}}}{\Gamma\left(\alpha_{1}+\alpha_{2}+\alpha_{3}+1\right)} \\
& +\frac{2 K^{\alpha_{1}+2 \alpha_{2}+2 \alpha_{3}}}{|\Delta| \Gamma\left(\alpha_{3}+1\right) \Gamma\left(\alpha_{2}+\alpha_{3}+1\right) \Gamma\left(\alpha_{1}+\alpha_{2}+\alpha_{3}-\alpha_{4}+1\right)} \\
& +\frac{K^{\alpha_{1}+2 \alpha_{2}+2 \alpha_{3}}}{|\Delta| \Gamma\left(\alpha_{3}+1\right) \Gamma\left(\alpha_{2}+\alpha_{3}-\alpha_{4}+1\right) \Gamma\left(\alpha_{1}+\alpha_{2}+\alpha_{3}+1\right)} \\
& +\frac{K^{\alpha_{1}+2 \alpha_{2}+2 \alpha_{3}}}{|\Delta| \Gamma\left(\alpha_{2}+\alpha_{3}+1\right) \Gamma\left(\alpha_{3}-\alpha_{4}+1\right) \Gamma\left(\alpha_{1}+\alpha_{2}+\alpha_{3}+1\right)},
\end{aligned}
$$




$$
\begin{aligned}
\Upsilon_{2}= & \frac{\mu K^{\alpha_{3}}}{\Gamma\left(\alpha_{3}+1\right)} \quad \frac{3 \mu K^{\alpha_{2}+2 \alpha_{3}}}{|\Delta| \Gamma\left(\alpha_{3}+1\right) \Gamma\left(\alpha_{3}-\alpha_{4}+1\right) \Gamma\left(\alpha_{2}+\alpha_{3}+1\right)} \\
& +\frac{n \rho k^{\alpha_{2}+\alpha_{3}}}{|\Delta| \Gamma\left(\alpha_{2}+\alpha_{3}+1\right) \Gamma\left(\alpha_{3}-\alpha_{4}+1\right)}+\frac{n k^{\alpha_{2}+\alpha_{3}}}{|\Delta| \Gamma\left(\alpha_{3}+1\right) \Gamma\left(\alpha_{2}+\alpha_{3}-\alpha_{4}+1\right)} \\
+ & \frac{\mu K^{\alpha_{2}+2 \alpha_{3}}}{|\Delta| \Gamma\left(\alpha_{3}+1\right) \Gamma\left(\alpha_{3}+1\right) \Gamma\left(\alpha_{2}+\alpha_{3}-\alpha_{4}+1\right)}, \\
\Upsilon_{3} \quad & \frac{K^{\alpha_{1}+\alpha_{2}+\alpha_{3}-\alpha_{4}}}{\Gamma\left(\alpha_{1}+\alpha_{2}+\alpha_{3}-\alpha_{4}+1\right)} \\
& +\frac{2 K^{\alpha_{1}+2 \alpha_{2}+2 \alpha_{3}-\alpha_{4}}}{|\Delta| \Gamma\left(\alpha_{3}-\alpha_{4}+1\right) \Gamma\left(\alpha_{2}+\alpha_{3}-\alpha_{4}+1\right) \Gamma\left(\alpha_{1}+\alpha_{2}+\alpha_{3}+1\right)} \\
& +\frac{K^{\alpha_{1}+2 \alpha_{2}+2 \alpha_{3}-\alpha_{4}}}{|\Delta| \Gamma\left(\alpha_{3}+1\right) \Gamma\left(\alpha_{2}+\alpha_{3}-\alpha_{4}+1\right) \Gamma\left(\alpha_{1}+\alpha_{2}+\alpha_{3}-\alpha_{4}+1\right)} \\
& +\frac{K^{\alpha_{1}+2 \alpha_{2}+2 \alpha_{3}-\alpha_{4}}}{|\Delta| \Gamma\left(\alpha_{3}-\alpha_{4}+1\right) \Gamma\left(\alpha_{2}+\alpha_{3}+1\right) \Gamma\left(\alpha_{1}+\alpha_{2}+\alpha_{3}-\alpha_{4}+1\right)},
\end{aligned}
$$

and

$$
\begin{aligned}
\Upsilon_{4}= & \frac{2 n \rho K^{\alpha_{2}+\alpha_{3}-\alpha_{4}}}{|\Delta| \Gamma\left(\alpha_{3}-\alpha_{4}+1\right) \Gamma\left(\alpha_{2}+\alpha_{3}-\alpha_{4}+1\right)}+\frac{\mu K^{\alpha_{3}-\alpha_{4}}}{\Gamma\left(\alpha_{3}-\alpha_{4}+1\right)} \\
& +\frac{3 \mu K^{\alpha_{2}+2 \alpha_{3}-\alpha_{4}}}{|\Delta| \Gamma\left(\alpha_{3}+1\right) \Gamma\left(\alpha_{3}-\alpha_{4}+1\right) \Gamma\left(\alpha_{2}+\alpha_{3}-\alpha_{4}+1\right)} \\
& +\frac{\mu K^{\alpha_{2}+2 \alpha_{3}-\alpha_{4}}}{|\Delta| \Gamma\left(\alpha_{3}-\alpha_{4}+1\right) \Gamma\left(\alpha_{3}-\alpha_{4}+1\right) \Gamma\left(\alpha_{2}+\alpha_{3}+1\right)}
\end{aligned}
$$

Proposition 1. Let $q>0$, and $\varphi, u, v \in C^{n}([a, b])$, we have

$$
\begin{gathered}
(\varphi(x)-\varphi(a))^{q} \leq k^{q}, \\
\left|I_{a^{+}}^{q ; \varphi} u(x)\right| \leq \frac{(\varphi(x)-\varphi(a))^{q}\|u\|}{\Gamma(q+1)}, \\
\left|g\left(s, u(s),{ }^{c} D_{a^{+}}^{\alpha_{4} ; \varphi} u(s)\right)\right| \leq \phi(t)+\rho_{1}\|u\|^{\delta_{1}}+\rho_{2}\|u\|^{\delta_{2}}, \\
\left|g\left(s, u(s),{ }^{c} D_{a^{+}}^{\alpha_{4} ; \varphi} u(s)\right)-g\left(s, v(s),{ }^{c} D_{a^{+}}^{\alpha_{4} ; \varphi} v(s)\right)\right| \leq \delta\|u-v\|
\end{gathered}
$$

and

$$
\left|I_{a^{+}}^{q ; \varphi} u(x)-I_{a^{+}}^{q ; \varphi} v(x)\right| \leq \frac{(\varphi(x)-\varphi(a))^{q}\|u-v\|}{\Gamma(q+1)}
$$

Proof. We have $\varphi$ is increasing and $a<x<b$, then

$$
(\varphi(x)-\varphi(a))^{q} \leq(\varphi(b)-\varphi(a))^{q}=k^{q},
$$




$$
\begin{aligned}
\left|I_{a^{+}}^{q ; \varphi} u(x)\right| & \leq \frac{1}{\Gamma(q)} \int_{a}^{t} \varphi^{\prime}(s)(\varphi(t)-\varphi(s))^{q-1}|u(s)| d s \\
& \leq \frac{\max _{s \in[a, b]}|u(s)|}{\Gamma(q)} \int_{a}^{t} \varphi^{\prime}(s)(\varphi(t)-\varphi(s))^{q-1} d s \\
& \leq \frac{(\varphi(x)-\varphi(a))^{q}\|u\|}{\Gamma(q+1)} .
\end{aligned}
$$

By $\left(\mathbb{H}_{3}\right)$, we have

$$
\begin{aligned}
\left|g\left(s, u(s),{ }^{c} D_{a^{+}}^{\alpha_{4} ; \varphi} u(s)\right)\right| & \leq \phi(t)+\rho_{1}|u(s)|^{\delta_{1}}+\rho_{2}\left|{ }^{c} D_{a^{+}}^{\alpha_{4} ; \varphi} u(s)\right|^{\delta_{2}} \\
& \leq \phi(t)+\rho_{1}\left(\max _{s \in[a, b]}|u(s)|\right)^{\delta_{1}}+\rho_{2}\left(\max _{s \in[a, b]}\left|{ }^{c} D_{a^{+}}^{\alpha_{4} ; \varphi} u(s)\right|\right)^{\delta_{2}} \\
& \leq \phi(t)+\rho_{1}\|u\|^{\delta_{1}}+\rho_{2}\|u\|^{\delta_{2}} .
\end{aligned}
$$

By $\left(\mathbb{H}_{2}\right)$, we have

$$
\begin{aligned}
& \left|g\left(s, u(s),{ }^{c} D_{a^{+}}^{\alpha_{4} ; \varphi} u(s)\right)-g\left(s, v(s),{ }^{c} D_{a^{+}}^{\alpha_{4} ; \varphi} v(s)\right)\right| \\
\leq & \delta\left(|u(s)-v(s)|+\left|{ }^{c} D_{a^{+}}^{\alpha_{4} ; \varphi} u(s)-{ }^{c} D_{a^{+}}^{\alpha_{4} ; \varphi} v(s)\right|\right) \\
\leq & \delta\left(|u(s)-v(s)|+\left|{ }^{c} D_{a^{+}}^{\alpha_{4} ; \varphi}(u(s)-v(s))\right|\right) \\
\leq & \delta\left(\max _{s \in[a, b]}|u(s)-v(s)|+\max _{s \in[a, b]}\left|{ }^{c} D_{a^{+}}^{\alpha_{4} ; \varphi}(u(s)-v(s))\right|\right) \\
\leq & \delta\|u-v\| .
\end{aligned}
$$

Theorem 1. Under the hypotheses $\left(\mathbb{H}_{1}\right)$ and $\left(\mathbb{H}_{3}\right)$, the fractional Langevin equation (1.1) has a solution.

Proof. We define the operator $\mathbb{T}: \mathbb{E} \rightarrow \mathbb{E}$ as follow:

$$
\begin{aligned}
& (\mathbb{T} u)(t) \\
= & I_{a^{+}}^{\alpha_{1}+\alpha_{2}+\alpha_{3} ; \varphi} g_{u}(t)-\mu I_{a^{+}}^{\alpha_{3} ; \varphi} u(t) \\
& +\frac{\rho(\varphi(t)-\varphi(a))^{\alpha_{2}+\alpha_{3}} \sum_{i=1}^{n} u\left(\zeta_{i}\right)}{\Delta \Gamma\left(\alpha_{2}+\alpha_{3}+1\right) \Gamma\left(\alpha_{3}-\alpha_{4}+1\right)}-\frac{\rho K^{\alpha_{2}}(\varphi(t)-\varphi(a))^{\alpha_{3}} \sum_{i=1}^{n} u\left(\zeta_{i}\right)}{\Delta \Gamma\left(\alpha_{3}+1\right) \Gamma\left(\alpha_{2}+\alpha_{3}-\alpha_{4}+1\right)} \\
& +\frac{\mu K^{\alpha_{4}}\left(K^{\alpha_{2}}(\varphi(t)-\varphi(a))^{\alpha_{3}}-(\varphi(t)-\varphi(a))^{\alpha_{2}+\alpha_{3}}\right) I_{a^{+}}^{\alpha_{3}-\alpha_{4} ; \varphi} u(b)}{\Delta \Gamma\left(\alpha_{3}+1\right) \Gamma\left(\alpha_{2}+\alpha_{3}+1\right)} \\
& +\frac{\mu(\varphi(t)-\varphi(a))^{\alpha_{2}+\alpha_{3}} I_{a^{+}}^{\alpha_{3} ; \varphi} u(b)}{\Delta \Gamma\left(\alpha_{2}+\alpha_{3}+1\right) \Gamma\left(\alpha_{3}-\alpha_{4}+1\right)}-\frac{\mu K^{\alpha_{2}}(\varphi(t)-\varphi(a))^{\alpha_{3}} I_{a^{+}}^{\alpha_{3} ; \varphi} u(b)}{\Delta \Gamma\left(\alpha_{3}+1\right) \Gamma\left(\alpha_{2}+\alpha_{3}-\alpha_{4}+1\right)} \\
+ & \frac{K^{\alpha_{4}}\left((\varphi(t)-\varphi(a))^{\alpha_{2}+\alpha_{3}}-K^{\alpha_{2}}(\varphi(t)-\varphi(a))^{\alpha_{3}}\right) I_{a^{+}}^{\alpha_{1}+\alpha_{2}+\alpha_{3}-\alpha_{4} ; \varphi} g_{u}(b)}{\Delta \Gamma\left(\alpha_{3}+1\right) \Gamma\left(\alpha_{2}+\alpha_{3}+1\right)} \\
+ & \frac{K^{\alpha_{2}}(\varphi(t)-\varphi(a))^{\alpha_{3}} I_{a^{+}}^{\alpha_{1}+\alpha_{2}+\alpha_{3} ; \varphi} g_{u}(b)}{\Delta \Gamma\left(\alpha_{3}+1\right) \Gamma\left(\alpha_{2}+\alpha_{3}-\alpha_{4}+1\right)}-\frac{(\varphi(t)-\varphi(a))^{\alpha_{2}+\alpha_{3}} I_{a^{+}}^{\alpha_{1}+\alpha_{2}+\alpha_{3} ; \varphi} g_{u}(b)}{\Delta \Gamma\left(\alpha_{2}+\alpha_{3}+1\right) \Gamma\left(\alpha_{3}-\alpha_{4}+1\right)}
\end{aligned}
$$

also

$$
{ }^{c} D_{a^{+}}^{\alpha_{4} ; \varphi}(\mathbb{T} u)(t)
$$




$$
\begin{aligned}
= & I_{a^{+}}^{\alpha_{1}+\alpha_{2}+\alpha_{3}-\alpha_{4} ; \varphi} g_{u}(t)-\mu I_{a^{+}}^{\alpha_{3}-\alpha_{4} ; \varphi} u(t) \\
& +\frac{u(b)\left((\varphi(t)-\varphi(a))^{\alpha_{2}+\alpha_{3}-\alpha_{4}}-K^{\alpha_{2}}(\varphi(t)-\varphi(a))^{\alpha_{3}-\alpha_{4}}\right)}{\Delta \Gamma\left(\alpha_{3}-\alpha_{4}+1\right) \Gamma\left(\alpha_{2}+\alpha_{3}-\alpha_{4}+1\right)} \\
& +\frac{\mu\left((\varphi(t)-\varphi(a))^{\alpha_{2}+\alpha_{3}-\alpha_{4}}-K^{\alpha_{2}}(\varphi(t)-\varphi(a))^{\alpha_{3}-\alpha_{4}}\right) I_{a^{+}}^{\alpha_{3} ; \varphi} u(b)}{\Delta \Gamma\left(\alpha_{3}-\alpha_{4}+1\right) \Gamma\left(\alpha_{2}+\alpha_{3}-\alpha_{4}+1\right)} \\
& +\frac{\mu K^{\alpha_{2}+\alpha_{4}}(\varphi(t)-\varphi(a))^{\alpha_{3}-\alpha_{4}} I_{a^{+}}^{\alpha_{3}-\alpha_{4} ; \varphi} u(b)}{\Delta \Gamma\left(\alpha_{3}-\alpha_{4}+1\right) \Gamma\left(\alpha_{2}+\alpha_{3}+1\right)} \\
& -\frac{K^{\alpha_{2}+\alpha_{4}}(\varphi(t)-\varphi(a))^{\alpha_{3}-\alpha_{4}} I_{a^{+}}^{\alpha_{1}+\alpha_{2}+\alpha_{3}-\alpha_{4} ; \varphi} g_{u}(b)}{\Delta \Gamma\left(\alpha_{3}-\alpha_{4}+1\right) \Gamma\left(\alpha_{2}+\alpha_{3}+1\right)} \\
& +\frac{\left(K^{\alpha_{2}}(\varphi(t)-\varphi(a))^{\alpha_{3}-\alpha_{4}}-(\varphi(t)-\varphi(a))^{\alpha_{2}+\alpha_{3}-\alpha_{4}}\right) I_{a^{+} g}^{\alpha_{1}+\alpha_{2}+\alpha_{3} ; \varphi} g_{u}(b)}{\Delta \Gamma\left(\alpha_{3}-\alpha_{4}+1\right) \Gamma\left(\alpha_{2}+\alpha_{3}-\alpha_{4}+1\right)} \\
& +\frac{K^{\alpha_{4}}(\varphi(t)-\varphi(a))^{\alpha_{2}+\alpha_{3}-\alpha_{4}} I_{a^{+}}^{\alpha_{1}+\alpha_{2}+\alpha_{3}-\alpha_{4} ; \varphi} g_{u}(b)}{\Delta \Gamma\left(\alpha_{3}+1\right) \Gamma\left(\alpha_{2}+\alpha_{3}-\alpha_{4}+1\right)} \\
& -\frac{\mu K^{\alpha_{4}}(\varphi(t)-\varphi(a))^{\alpha_{2}+\alpha_{3}-\alpha_{4}} I_{a^{+}}^{\alpha_{3}-\alpha_{4} ; \varphi} u(b)}{\Delta \Gamma\left(\alpha_{3}+1\right) \Gamma\left(\alpha_{2}+\alpha_{3}-\alpha_{4}+1\right)}
\end{aligned}
$$

where if $q \in\left\{\alpha_{3}, \alpha_{3}-\alpha_{4}, \alpha_{1}+\alpha_{2}+\alpha_{3}, \alpha_{1}+\alpha_{2}+\alpha_{3}-\alpha_{4}\right\}, x \in\{t, b\}$, then

$$
I_{a^{+}}^{q ; \varphi} u(x)=\frac{1}{\Gamma(q)} \int_{a}^{x} \varphi^{\prime}(s)(\varphi(x)-\varphi(s))^{q-1} u(s) d s,
$$

and

$$
I_{a^{+}}^{q ; \varphi} g_{u}(x)=\frac{1}{\Gamma(q)} \int_{a}^{x} \varphi^{\prime}(s)(\varphi(x)-\varphi(s))^{q-1} g\left(s, u(s),{ }^{c} D_{a^{+}}^{\alpha_{4} ; \varphi} u(s)\right) d s .
$$

Lemma 2 implies that the fixed points of the operator $\mathbb{T}$ are the same solutions of the boundary value problem (1.1). We consider a ball $\mathbb{U}_{r}=\{u \in \mathbb{E},\|u\| \leq r\}$ so that

$$
\max \left\{4\left(\Upsilon_{1}+\Upsilon_{3}\right)\|\phi\|,\left(4\left(\Upsilon_{1}+\Upsilon_{3}\right) \rho_{1}\right)^{\frac{1}{1-\delta_{1}}},\left(4\left(\Upsilon_{1}+\Upsilon_{3}\right) \rho_{2}\right)^{\frac{1}{1-\delta_{2}}}, 4\left(\Upsilon_{2}+\Upsilon_{4}\right)\right\} \leq r
$$

For any $u \in \mathbb{U}_{r}$ and by $\left(\mathbb{H}_{3}\right)$, we show that $\mathbb{T} \mathbb{U}_{r} \subset \mathbb{U}_{r}$, then

$$
\begin{aligned}
& |(\mathbb{T} u)(t)| \\
\leq & \left|I_{a^{+}}^{\alpha_{1}+\alpha_{2}+\alpha_{3} ; \varphi} g_{u}(t)\right|+\mu\left|I_{a^{+}}^{\alpha_{3} ; \varphi} u(t)\right| \\
& +\frac{\rho(\varphi(t)-\varphi(a))^{\alpha_{2}+\alpha_{3}} \sum_{i=1}^{n}\left|u\left(\zeta_{i}\right)\right|}{|\Delta| \Gamma\left(\alpha_{2}+\alpha_{3}+1\right) \Gamma\left(\alpha_{3}-\alpha_{4}+1\right)}+\frac{\rho K^{\alpha_{2}}(\varphi(t)-\varphi(a))^{\alpha_{3}} \sum_{i=1}^{n}\left|u\left(\zeta_{i}\right)\right|}{|\Delta| \Gamma\left(\alpha_{3}+1\right) \Gamma\left(\alpha_{2}+\alpha_{3}-\alpha_{4}+1\right)} \\
& +\frac{\mu K^{\alpha_{4}}\left(K^{\alpha_{2}}(\varphi(t)-\varphi(a))^{\alpha_{3}}+(\varphi(t)-\varphi(a))^{\alpha_{2}+\alpha_{3}}\right)\left|I_{a^{+}}^{\alpha_{3}-\alpha_{4} ; \varphi} u(b)\right|}{|\Delta| \Gamma\left(\alpha_{3}+1\right) \Gamma\left(\alpha_{2}+\alpha_{3}+1\right)} \\
& +\frac{\mu(\varphi(t)-\varphi(a))^{\alpha_{2}+\alpha_{3}}\left|I_{a^{+}}^{\alpha_{3} ; \varphi} u(b)\right|}{|\Delta| \Gamma\left(\alpha_{2}+\alpha_{3}+1\right) \Gamma\left(\alpha_{3}-\alpha_{4}+1\right)}+\frac{\mu K^{\alpha_{2}}(\varphi(t)-\varphi(a))^{\alpha_{3}}\left|I_{a^{+}}^{\alpha_{3} ; \varphi} u(b)\right|}{|\Delta| \Gamma\left(\alpha_{3}+1\right) \Gamma\left(\alpha_{2}+\alpha_{3}-\alpha_{4}+1\right)} \\
& +\frac{K^{\alpha_{4}}\left((\varphi(t)-\varphi(a))^{\alpha_{2}+\alpha_{3}}+K^{\alpha_{2}}(\varphi(t)-\varphi(a))^{\alpha_{3}}\right)\left|I_{a^{+}}^{\alpha_{1}+\alpha_{2}+\alpha_{3}-\alpha_{4} ; \varphi} g_{u}(b)\right|}{|\Delta| \Gamma\left(\alpha_{3}+1\right) \Gamma\left(\alpha_{2}+\alpha_{3}+1\right)} \\
& +\frac{K^{\alpha_{2}}(\varphi(t)-\varphi(a))^{\alpha_{3}}\left|I_{a^{+}}^{\alpha_{1}+\alpha_{2}+\alpha_{3} ; \varphi} g_{u}(b)\right|}{|\Delta| \Gamma\left(\alpha_{3}+1\right) \Gamma\left(\alpha_{2}+\alpha_{3}-\alpha_{4}+1\right)}+\frac{(\varphi(t)-\varphi(a))^{\alpha_{2}+\alpha_{3}}\left|I_{a^{+}}^{\alpha_{1}+\alpha_{2}+\alpha_{3} ; \varphi} g_{u}(b)\right|}{|\Delta| \Gamma\left(\alpha_{2}+\alpha_{3}+1\right) \Gamma\left(\alpha_{3}-\alpha_{4}+1\right)},
\end{aligned}
$$


by Lemma 4, and Proposition 1, we obtain

$$
\begin{aligned}
|(\mathbb{T} u)(t)| \leq & \left(\|\phi\|+\rho_{1} r^{\delta_{1}}+\rho_{3} r^{\delta_{2}}\right)\left\{\frac{K^{\alpha_{1}+\alpha_{2}+\alpha_{3}}}{\Gamma\left(\alpha_{1}+\alpha_{2}+\alpha_{3}+1\right)}\right. \\
& +\frac{2 K^{\alpha_{1}+2 \alpha_{2}+2 \alpha_{3}}}{|\Delta| \Gamma\left(\alpha_{3}+1\right) \Gamma\left(\alpha_{2}+\alpha_{3}+1\right) \Gamma\left(\alpha_{1}+\alpha_{2}+\alpha_{3}-\alpha_{4}+1\right)} \\
& +\frac{K^{\alpha_{1}+2 \alpha_{2}+2 \alpha_{3}}}{|\Delta| \Gamma\left(\alpha_{3}+1\right) \Gamma\left(\alpha_{2}+\alpha_{3}-\alpha_{4}+1\right) \Gamma\left(\alpha_{1}+\alpha_{2}+\alpha_{3}+1\right)} \\
& \left.+\frac{K^{\alpha_{1}+2 \alpha_{2}+2 \alpha_{3}}}{|\Delta| \Gamma\left(\alpha_{2}+\alpha_{3}+1\right) \Gamma\left(\alpha_{3}-\alpha_{4}+1\right) \Gamma\left(\alpha_{1}+\alpha_{2}+\alpha_{3}+1\right)}\right\} \\
& +r\left\{\frac{\mu K^{\alpha_{3}}}{\Gamma\left(\alpha_{3}+1\right)}+\frac{3 \mu K^{\alpha_{2}+2 \alpha_{3}}}{|\Delta| \Gamma\left(\alpha_{3}+1\right) \Gamma\left(\alpha_{3}-\alpha_{4}+1\right) \Gamma\left(\alpha_{2}+\alpha_{3}+1\right)} n \rho k^{\alpha_{2}+\alpha_{3}}\right. \\
& +\frac{n \rho k^{\alpha_{2}+\alpha_{3}}}{|\Delta| \Gamma\left(\alpha_{2}+\alpha_{3}+1\right) \Gamma\left(\alpha_{3}-\alpha_{4}+1\right)}+\frac{\left.\mu \alpha_{3}+\alpha_{3}+1\right) \Gamma\left(\alpha_{2}+\alpha_{3}-\alpha_{4}+1\right)}{|\Delta| \alpha_{2}+2 \alpha_{3}} \\
& \left.+\frac{\alpha_{1}}{|\Delta| \Gamma\left(\alpha_{3}+1\right) \Gamma\left(\alpha_{3}+1\right) \Gamma\left(\alpha_{2}+\alpha_{3}-\alpha_{4}+1\right)}\right\} \\
\leq & \Upsilon_{1}\left(\|\phi\|+\rho_{1} r^{\delta_{1}}+\rho_{3} r^{\delta_{2}}\right)+\Upsilon_{2} r .
\end{aligned}
$$

Also

$$
\begin{aligned}
& \left|{ }^{c} D_{a^{+}}^{\alpha_{4} ; \varphi}(\mathbb{T} u)(t)\right| \\
\leq & \left|I_{a^{+}}^{\alpha^{+}+\alpha_{2}+\alpha_{3}-\alpha_{4} ; \varphi} g_{u}(t)\right|+\mu\left|I_{a^{+}}^{\alpha_{3}-\alpha_{4} ; \varphi} u(t)\right| \\
& +\frac{u(b)\left((\varphi(t)-\varphi(a))^{\alpha_{2}+\alpha_{3}-\alpha_{4}}+K^{\alpha_{2}}(\varphi(t)-\varphi(a))^{\alpha_{3}-\alpha_{4}}\right)}{|\Delta| \Gamma\left(\alpha_{3}-\alpha_{4}+1\right) \Gamma\left(\alpha_{2}+\alpha_{3}-\alpha_{4}+1\right)} \\
& +\frac{\mu\left((\varphi(t)-\varphi(a))^{\alpha_{2}+\alpha_{3}-\alpha_{4}}+K^{\alpha_{2}}(\varphi(t)-\varphi(a))^{\alpha_{3}-\alpha_{4}}\right)\left|I_{a^{+}}^{\alpha_{3} ; \varphi} u(b)\right|}{|\Delta| \Gamma\left(\alpha_{3}-\alpha_{4}+1\right) \Gamma\left(\alpha_{2}+\alpha_{3}-\alpha_{4}+1\right)} \\
& +\frac{\mu K^{\alpha_{2}+\alpha_{4}}(\varphi(t)-\varphi(a))^{\alpha_{3}-\alpha_{4}}\left|I_{a^{+}}^{\alpha_{3}-\alpha_{4} ; \varphi} u(b)\right|}{|\Delta| \Gamma\left(\alpha_{3}-\alpha_{4}+1\right) \Gamma\left(\alpha_{2}+\alpha_{3}+1\right)} \\
& +\frac{\mu K^{\alpha_{4}}(\varphi(t)-\varphi(a))^{\alpha_{2}+\alpha_{3}-\alpha_{4}}\left|I_{a^{+}}^{\alpha_{3}-\alpha_{4} ; \varphi} u(b)\right|}{|\Delta| \Gamma\left(\alpha_{3}+1\right) \Gamma\left(\alpha_{2}+\alpha_{3}-\alpha_{4}+1\right)} \\
& +\frac{\left(K^{\alpha_{2}}(\varphi(t)-\varphi(a))^{\alpha_{3}-\alpha_{4}}+(\varphi(t)-\varphi(a))^{\alpha_{2}+\alpha_{3}-\alpha_{4}}\right)\left|I_{a^{+}}^{\alpha_{1}+\alpha_{2}+\alpha_{3} ; \varphi} g_{u}(b)\right|}{|\Delta| \Gamma\left(\alpha_{3}-\alpha_{4}+1\right) \Gamma\left(\alpha_{2}+\alpha_{3}-\alpha_{4}+1\right)} \\
& +\frac{K^{\alpha_{4}}(\varphi(t)-\varphi(a))^{\alpha_{2}+\alpha_{3}-\alpha_{4}}\left|I_{a^{+}}^{\alpha_{1}+\alpha_{2}+\alpha_{3}-\alpha_{4} ; \varphi} g_{u}(b)\right|}{|\Delta| \Gamma\left(\alpha_{3}+1\right) \Gamma\left(\alpha_{2}+\alpha_{3}-\alpha_{4}+1\right)} \\
& +\frac{K^{\alpha_{2}+\alpha_{4}}(\varphi(t)-\varphi(a))^{\alpha_{3}-\alpha_{4}}\left|I_{a^{+}}^{\alpha_{1}+\alpha_{2}+\alpha_{3}-\alpha_{4} ; \varphi} g_{u}(b)\right|}{|\Delta| \Gamma\left(\alpha_{3}-\alpha_{4}+1\right) \Gamma\left(\alpha_{2}+\alpha_{3}+1\right)},
\end{aligned}
$$

by Lemma 4, and Proposition 1, we obtain

$$
\begin{aligned}
& \left|{ }^{c} D_{a^{+}}^{\alpha_{4} ; \varphi}(\mathbb{T} u)(t)\right| \\
\leq & \left(\|\phi\|+\rho_{1} r^{\delta_{1}}+\rho_{3} r^{\delta_{2}}\right)\left\{\frac{K^{\alpha_{1}+\alpha_{2}+\alpha_{3}-\alpha_{4}}}{\Gamma\left(\alpha_{1}+\alpha_{2}+\alpha_{3}-\alpha_{4}+1\right)}\right. \\
& +\frac{2 K^{\alpha_{1}+2 \alpha_{2}+2 \alpha_{3}-\alpha_{4}}}{|\Delta| \Gamma\left(\alpha_{3}-\alpha_{4}+1\right) \Gamma\left(\alpha_{2}+\alpha_{3}-\alpha_{4}+1\right) \Gamma\left(\alpha_{1}+\alpha_{2}+\alpha_{3}+1\right)} \\
& +\frac{K^{\alpha_{1}+2 \alpha_{2}+2 \alpha_{3}-\alpha_{4}}}{|\Delta| \Gamma\left(\alpha_{3}+1\right) \Gamma\left(\alpha_{2}+\alpha_{3}-\alpha_{4}+1\right) \Gamma\left(\alpha_{1}+\alpha_{2}+\alpha_{3}-\alpha_{4}+1\right)}
\end{aligned}
$$




$$
\begin{aligned}
& \left.+\frac{K^{\alpha_{1}+2 \alpha_{2}+2 \alpha_{3}-\alpha_{4}}}{|\Delta| \Gamma\left(\alpha_{3}-\alpha_{4}+1\right) \Gamma\left(\alpha_{2}+\alpha_{3}+1\right) \Gamma\left(\alpha_{1}+\alpha_{2}+\alpha_{3}-\alpha_{4}+1\right)}\right\} \\
& +r\left\{\frac{\mu n \rho K^{\alpha_{2}+\alpha_{3}-\alpha_{4}}}{|\Delta| \Gamma\left(\alpha_{3}-\alpha_{4}+1\right) \Gamma\left(\alpha_{2}+\alpha_{3}-\alpha_{4}+1\right)}+\frac{\mu K^{\alpha_{3}-\alpha_{4}}}{\Gamma\left(\alpha_{3}-\alpha_{4}+1\right)}\right. \\
& +\frac{3 \mu K^{\alpha_{2}+2 \alpha_{3}-\alpha_{4}}}{|\Delta| \Gamma\left(\alpha_{3}+1\right) \Gamma\left(\alpha_{3}-\alpha_{4}+1\right) \Gamma\left(\alpha_{2}+\alpha_{3}-\alpha_{4}+1\right)} \\
& \left.+\frac{\mu K^{\alpha_{2}+2 \alpha_{3}-\alpha_{4}}}{|\Delta| \Gamma\left(\alpha_{3}-\alpha_{4}+1\right) \Gamma\left(\alpha_{3}-\alpha_{4}+1\right) \Gamma\left(\alpha_{2}+\alpha_{3}+1\right)}\right\} \\
& \leq \Upsilon_{3}\left(\|\phi\|+\rho_{1} r^{\delta_{1}}+\rho_{3} r^{\delta_{2}}\right)+\Upsilon_{4} r .
\end{aligned}
$$

So

$$
\begin{aligned}
\|\mathbb{T} u\| & =\max |\mathbb{T} u(t)|+\max \left|{ }^{c} D_{a^{+}}^{\alpha_{4} ; \varphi}(\mathbb{T} u)(t)\right| \\
& \leq\left(\Upsilon_{1}+\Upsilon_{3}\right)\left(\|\phi\|+\rho_{1} r^{\delta_{1}}+\rho_{3} r^{\delta_{2}}\right)+\left(\Upsilon_{2}+\Upsilon_{4}\right) r \\
& \leq \frac{r}{4}+\frac{r}{4}+\frac{r}{4}+\frac{r}{4}=r .
\end{aligned}
$$

Next, we prove that the operator $\mathbb{T}$ is completely continuous. The functions $\varphi, u, g$ are continuous, hence the operator $\mathbb{T}$ is continuous. For any $u \in \mathbb{U}_{r}$ and $t_{1}, t_{2} \in[a, b]$ such that $t_{1}<t_{2}$, by Proposition 1 , Lemma 5 we have

$$
\begin{aligned}
& \left|(\mathbb{T} u)\left(t_{2}\right)-(\mathbb{T} u)\left(t_{1}\right)\right| \\
\leq & \frac{2\left(\|\phi\|+\rho_{1} r^{\delta_{1}}+\rho_{3} r^{\delta_{2}}\right)}{\Gamma\left(\alpha_{1}+\alpha_{2}+\alpha_{3}+1\right)}\left(\varphi\left(t_{2}\right)-\varphi\left(t_{1}\right)\right)^{\alpha_{1}+\alpha_{2}+\alpha_{3}} \\
& +\frac{2\left(\varphi\left(t_{2}\right)-\varphi\left(t_{1}\right)\right)^{\alpha_{3}}}{|\Delta| \Gamma\left(\alpha_{3}+1\right)}\left\{\mu r|\Delta|+\frac{\mu r K^{\alpha_{3}+\alpha_{2}}}{\Gamma\left(\alpha_{3}-\alpha_{4}+1\right) \Gamma\left(\alpha_{2}+\alpha_{3}+1\right)}\right. \\
& +\frac{\mu r K^{\alpha_{2}+\alpha_{3}}}{\Gamma\left(\alpha_{3}+1\right) \Gamma\left(\alpha_{2}+\alpha_{3}-\alpha_{4}+1\right)}+\frac{K^{\alpha_{1}+2 \alpha_{2}+\alpha_{3}-\alpha_{4}}\left(\|\phi\|+\rho_{1} r^{\delta_{1}}+\rho_{3} r^{\delta_{2}}\right)}{\Gamma\left(\alpha_{2}+\alpha_{3}+1\right) \Gamma\left(\alpha_{1}+\alpha_{2}+\alpha_{3}-\alpha_{4}+1\right)} \\
& \left.+\frac{K^{\alpha_{1}+2 \alpha_{2}+\alpha_{3}}\left(\|\phi\|+\rho_{1} r^{\delta_{1}}+\rho_{3} r^{\delta_{2}}\right)}{\Gamma\left(\alpha_{2}+\alpha_{3}-\alpha_{4}+1\right) \Gamma\left(\alpha_{1}+\alpha_{2}+\alpha_{3}+1\right)}+\frac{\rho n r K^{\alpha_{2}}}{\Gamma\left(\alpha_{2}+\alpha_{3}-\alpha_{4}+1\right)}\right\} \\
& +\frac{2\left(\varphi\left(t_{2}\right)-\varphi\left(t_{1}\right)\right)^{\alpha_{2}+\alpha_{3}}}{|\Delta| \Gamma\left(\alpha_{2}+\alpha_{3}+1\right)}\left\{\frac{\rho r K^{\alpha_{3}}}{\Gamma\left(\alpha_{3}-\alpha_{4}+1\right)}+\frac{K^{\alpha_{1}+\alpha_{2}+\alpha_{3}}}{\Gamma\left(\alpha_{3}+1\right) \Gamma\left(\alpha_{3}-\alpha_{4}+1\right)}\right. \\
& +\frac{\mu r K^{\alpha_{3}}}{\Gamma\left(\alpha_{3}+1\right) \Gamma\left(\alpha_{3}-\alpha_{4} r^{\delta_{1}}+\rho_{3} r^{\delta_{2}}\right)} \\
& \left.+\frac{K^{\alpha_{1}+\alpha_{2}+\alpha_{3}}\left(\|\phi\|+\rho_{1} r^{\delta_{1}}+\rho_{3} r^{\delta_{2}}\right)}{\Gamma\left(\alpha_{3}-\alpha_{4}+1\right) \Gamma\left(\alpha_{1}+\alpha_{2}+\alpha_{3}+1\right)}\right\} .
\end{aligned}
$$

Also

$$
\begin{aligned}
& \left|{ }^{c} D_{a^{+}}^{\alpha_{4} ; \varphi}(\mathbb{T} u)\left(t_{2}\right)-{ }^{c} D_{a^{+}}^{\alpha_{4} ; \varphi}(\mathbb{T} u)\left(t_{2}\right)\right| \\
\leq & \frac{2\left(\|\phi\|+\rho_{1} r^{\delta_{1}}+\rho_{3} r^{\delta_{2}}\right)}{\Gamma\left(\alpha_{1}+\alpha_{2}+\alpha_{3}-\alpha_{4}+1\right)}\left(\varphi\left(t_{2}\right)-\varphi\left(t_{1}\right)\right)^{\alpha_{1}+\alpha_{2}+\alpha_{3}-\alpha_{4}} \\
& +\frac{2\left(\varphi\left(t_{2}\right)-\varphi\left(t_{1}\right)\right)^{\alpha_{3}-\alpha_{4}}}{|\Delta|}\left\{\mu r|\Delta|+\frac{K^{\alpha_{1}+2 \alpha_{2}+\alpha_{3}}\left(\|\phi\|+\rho_{1} r^{\delta_{1}}+\rho_{3} r^{\delta_{2}}\right)}{\Gamma\left(\alpha_{2}+\alpha_{3}+1\right) \Gamma\left(\alpha_{1}+\alpha_{2}+\alpha_{3}-\alpha_{4}+1\right)}\right. \\
& +\frac{n r \rho K^{\alpha_{2}}}{\Gamma\left(\alpha_{2}+\alpha_{3}-\alpha_{4}+1\right)}+\frac{K^{\alpha_{1}+2 \alpha_{2}+\alpha_{3}}\left(\|\phi\|+\rho_{1} r^{\delta_{1}}+\rho_{3} r^{\delta_{2}}\right)}{\Gamma\left(\alpha_{2}+\alpha_{3}-\alpha_{4}+1\right) \Gamma\left(\alpha_{1}+\alpha_{2}+\alpha_{3}+1\right)}
\end{aligned}
$$




$$
\begin{aligned}
& \left.+\frac{\mu r K^{\alpha_{2}+\alpha_{3}}}{\Gamma\left(\alpha_{2}+\alpha_{3}-\alpha_{4}+1\right)}+\frac{\mu r K^{\alpha_{2}+\alpha_{3}}}{\Gamma\left(\alpha_{3}-\alpha_{4}+1\right) \Gamma\left(\alpha_{2}+\alpha_{3}+1\right)}\right\} \\
& +\frac{2\left(\varphi\left(t_{2}\right)-\varphi\left(t_{1}\right)\right)^{\alpha_{2}+\alpha_{3}-\alpha_{4}}}{|\Delta| \Gamma\left(\alpha_{2}+\alpha_{3}-\alpha_{4}+1\right)}\left\{\frac{\mu r K^{\alpha_{3}}}{\Gamma\left(\alpha_{3}+1\right) \Gamma\left(\alpha_{3}-\alpha_{4}+1\right)}\right. \\
& +\frac{n r \rho}{\Gamma\left(\alpha_{3}-\alpha_{4}+1\right)}+\frac{K^{\alpha_{1}+\alpha_{2}+\alpha_{3}}\left(\|\phi\|+\rho_{1} r^{\delta_{1}}+\rho_{3} r^{\delta_{2}}\right)}{\Gamma\left(\alpha_{3}-\alpha_{4}+1\right) \Gamma\left(\alpha_{1}+\alpha_{2}+\alpha_{3}+1\right)} \\
& \left.+\frac{\mu r K^{\alpha_{3}}}{\Gamma\left(\alpha_{3}-\alpha_{4}+1\right)}+\frac{K^{\alpha_{1}+\alpha_{2}+\alpha_{3}}\left(\|\phi\|+\rho_{1} r^{\delta_{1}}+\rho_{3} r^{\delta_{2}}\right)}{\Gamma\left(\alpha_{3}+1\right) \Gamma\left(\alpha_{1}+\alpha_{2}+\alpha_{3}-\alpha_{4}+1\right)}\right\}
\end{aligned}
$$

So (3.7) and (3.8) are independent of $u$ and close to zero when letting $t_{2} \rightarrow t_{1}$. Then, $\mathbb{T}\left(\mathbb{U}_{r}\right)$ is equicontinuous and the Arzela-Ascoli theorem implies that $\overline{\mathbb{T}\left(\mathbb{U}_{r}\right)}$ is compact, hence the operator $\mathbb{T}: \mathbb{U}_{r} \rightarrow \mathbb{U}_{r}$ is completely continuous. Therefore, by the Schauder fixed-point theorem, we conclude that the problem (1.1) has a solution.

By applying Banach fixed point theorem, we prove the uniqueness of solution of the problem (1.1).

Theorem 2. Let the assumptions $\left(\mathbb{H}_{1}-\mathbb{H}_{3}\right)$ are satisfied, then the boundary value problem (1.1) has a uniqueness solution provided that $\eta=\eta_{1}+\eta_{2}<1$, where

$$
\begin{aligned}
\eta_{1}= & \frac{\delta K^{\alpha_{1}+\alpha_{2}+\alpha_{3}}}{\Gamma\left(\alpha_{1}+\alpha_{2}+\alpha_{3}+1\right)}+\frac{2 \mu K^{\alpha_{2}+2 \alpha_{3}}}{|\Delta| \Gamma\left(\alpha_{3}+1\right) \Gamma\left(\alpha_{2}+\alpha_{3}+1\right)} \\
& +\frac{\mu K^{\alpha_{3}}}{\Gamma\left(\alpha_{3}+1\right)}+\frac{\mu K^{\alpha_{2}+2 \alpha_{3}}}{|\Delta| \Gamma\left(\alpha_{3}+1\right) \Gamma\left(\alpha_{3}+1\right) \Gamma\left(\alpha_{2}+\alpha_{3}-\alpha_{4}+1\right)} \\
& +\frac{\rho n K^{\alpha_{2}+\alpha_{3}}}{|\Delta| \Gamma\left(\alpha_{2}+\alpha_{3}+1\right) \Gamma\left(\alpha_{3}-\alpha_{4}+1\right)}+\frac{\rho K_{2}+\alpha_{3}}{|\Delta| \Gamma\left(\alpha_{3}+1\right) \Gamma\left(\alpha_{2}+\alpha_{3}-\alpha_{4}+1\right)} \\
& +\frac{\delta K^{\alpha_{2}+2 \alpha_{3}}}{|\Delta| \Gamma\left(\alpha_{2}+\alpha_{3}+1\right) \Gamma\left(\alpha_{3}+1\right) \Gamma\left(\alpha_{3}-\alpha_{4}+1\right)} \\
& +\frac{\delta K^{\alpha_{1}+2 \alpha_{3}+\alpha_{4}}}{|\Delta| \Gamma\left(\alpha_{3}+1\right) \Gamma\left(\alpha_{2}+\alpha_{3}+1\right) \Gamma\left(\alpha_{1}+\alpha_{2}+\alpha_{3}+1\right)} \\
& +\frac{\delta K^{\alpha_{1}+2 \alpha_{2}+2 \alpha_{3}}}{|\Delta| \Gamma\left(\alpha_{3}+1\right) \Gamma\left(\alpha_{2}+\alpha_{3}-\alpha_{4}+1\right) \Gamma\left(\alpha_{1}+\alpha_{2}+\alpha_{3}+1\right)}
\end{aligned}
$$

and

$$
\begin{aligned}
\eta_{2}= & \frac{\delta K^{\alpha_{1}+\alpha_{2}+\alpha_{3}-\alpha_{4}}}{\Gamma\left(\alpha_{1}+\alpha_{2}+\alpha_{3}-\alpha_{4}+1\right)}+\frac{\mu K^{\alpha_{3}-\alpha_{4}}}{\Gamma\left(\alpha_{3}-\alpha_{4}+1\right)} \\
& +\frac{2 n \rho K^{\alpha_{2}+\alpha_{3}-\alpha_{4}}}{|\Delta| \Gamma\left(\alpha_{3}-\alpha_{4}+1\right) \Gamma\left(\alpha_{2}+\alpha_{3}-\alpha_{4}+1\right)} \\
& +\frac{3 \mu K^{\alpha_{2}+2 \alpha_{3}-\alpha_{4}}}{|\Delta| \Gamma\left(\alpha_{3}+1\right) \Gamma\left(\alpha_{3}-\alpha_{4}+1\right) \Gamma\left(\alpha_{2}+\alpha_{3}-\alpha_{4}+1\right)} \\
& +\frac{\mu K^{\alpha_{2}+2 \alpha_{3}-\alpha_{4}}}{|\Delta| \Gamma\left(\alpha_{3}-\alpha_{4}+1\right) \Gamma\left(\alpha_{3}-\alpha_{4}+1\right) \Gamma\left(\alpha_{2}+\alpha_{3}+1\right)} \\
& +\frac{2 \delta K^{\alpha_{1}+2 \alpha_{2}+2 \alpha_{3}-\alpha_{4}}}{|\Delta| \Gamma\left(\alpha_{3}-\alpha_{4}+1\right) \Gamma\left(\alpha_{2}+\alpha_{3}-\alpha_{4}+1\right) \Gamma\left(\alpha_{1}+\alpha_{2}+\alpha_{3}+1\right)}
\end{aligned}
$$




$$
\begin{aligned}
& +\frac{\delta K^{\alpha_{1}+2 \alpha_{2}+2 \alpha_{3}-\alpha_{4}}}{|\Delta| \Gamma\left(\alpha_{3}+1\right) \Gamma\left(\alpha_{2}+\alpha_{3}-\alpha_{4}+1\right) \Gamma\left(\alpha_{1}+\alpha_{2}+\alpha_{3}-\alpha_{4}+1\right)} \\
& +\frac{\delta K^{\alpha_{1}+2 \alpha_{2}+2 \alpha_{3}-\alpha_{4}}}{|\Delta| \Gamma\left(\alpha_{3}-\alpha_{4}+1\right) \Gamma\left(\alpha_{2}+\alpha_{3}+1\right) \Gamma\left(\alpha_{1}+\alpha_{2}+\alpha_{3}-\alpha_{4}+1\right)}
\end{aligned}
$$

Proof. For any $u, v \in \mathbb{U}, t \in[a, b]$ and by condition $\left(\mathbb{H}_{2}\right)$, and proposition 1 we give

$$
\begin{aligned}
& |(\mathbb{T} u)(t)-(\mathbb{T} v)(t)| \\
\leq & \left|I_{a^{+}}^{\alpha_{1}+\alpha_{2}+\alpha_{3} ; \varphi} g_{u}(t)-I_{a^{+}}^{\alpha_{1}+\alpha_{2}+\alpha_{3} ; \varphi} g_{v}(t)\right|+\mu\left|I_{a^{+}}^{\alpha_{3} ; \varphi} u(t)-I_{a^{+}}^{\alpha_{3} ; \varphi} v(t)\right| \\
& +\frac{\rho(\varphi(t)-\varphi(a))^{\alpha_{2}+\alpha_{3}} \sum_{i=1}^{n}\left|u\left(\zeta_{i}\right)-v\left(\zeta_{i}\right)\right|}{|\Delta| \Gamma\left(\alpha_{2}+\alpha_{3}+1\right) \Gamma\left(\alpha_{3}-\alpha_{4}+1\right)} \\
& +\frac{\rho K^{\alpha_{2}}(\varphi(t)-\varphi(a))^{\alpha_{3}} \sum_{i=1}^{n}\left|u\left(\zeta_{i}\right)-v\left(\zeta_{i}\right)\right|}{|\Delta| \Gamma\left(\alpha_{3}+1\right) \Gamma\left(\alpha_{2}+\alpha_{3}-\alpha_{4}+1\right)} \\
& +\frac{\mu K^{\alpha_{4}}\left(K^{\alpha_{2}}(\varphi(t)-\varphi(a))^{\alpha_{3}}+(\varphi(t)-\varphi(a))^{\alpha_{2}+\alpha_{3}}\right)\left|I_{a^{+}}^{\alpha_{3}-\alpha_{4} ; \varphi} u(b)-I_{a^{+}}^{\alpha_{3}-\alpha_{4} ; \varphi} v(b)\right|}{|\Delta| \Gamma\left(\alpha_{3}+1\right) \Gamma\left(\alpha_{2}+\alpha_{3}+1\right)} \\
+ & \frac{\mu(\varphi(t)-\varphi(a))^{\alpha_{2}+\alpha_{3}}\left|I_{a^{+}}^{\alpha_{3} ; \varphi} u(b)-I_{a^{+}}^{\alpha_{3} ; \varphi} v(b)\right|}{|\Delta| \Gamma\left(\alpha_{2}+\alpha_{3}+1\right) \Gamma\left(\alpha_{3}-\alpha_{4}+1\right)} \\
+ & \frac{\mu K^{\alpha_{2}}(\varphi(t)-\varphi(a))^{\alpha_{3}}\left|I_{a^{+}}^{\alpha_{3} ; \varphi} u(b)-I_{a^{+}}^{\alpha_{3} ; \varphi} v(b)\right|}{|\Delta| \Gamma\left(\alpha_{3}+1\right) \Gamma\left(\alpha_{2}+\alpha_{3}-\alpha_{4}+1\right)} \\
& +\frac{K^{\alpha_{4}}\left((\varphi(t)-\varphi(a))^{\alpha_{2}+\alpha_{3}}+K^{\alpha_{2}}(\varphi(t)-\varphi(a))^{\alpha_{3}}\right)\left|I_{a^{+}}^{\alpha_{1}+\alpha_{2}+\alpha_{3} ; \varphi} g_{u}(b)-I_{a^{+}}^{\alpha_{1}+\alpha_{2}+\alpha_{3} ; \varphi} g_{v}(b)\right|}{|\Delta| \Gamma\left(\alpha_{3}+1\right) \Gamma\left(\alpha_{2}+\alpha_{3}+1\right)} \\
+ & \frac{K^{\alpha_{2}}(\varphi(t)-\varphi(a))^{\alpha_{3}}\left|I_{a^{+}}^{\alpha_{1}+\alpha_{2}+\alpha_{3} ; \varphi} g_{u}(b)-I_{a^{+}}^{\alpha_{1}+\alpha_{2}+\alpha_{3} ; \varphi} g_{v}(b)\right|}{|\Delta| \Gamma\left(\alpha_{3}+1\right) \Gamma\left(\alpha_{2}+\alpha_{3}-\alpha_{4}+1\right)} \\
+ & \frac{(\varphi(t)-\varphi(a))^{\alpha_{2}+\alpha_{3}}\left|I_{a^{+}}^{\alpha_{1}+\alpha_{2}+\alpha_{3} ; \varphi} g_{u}(b)-I_{a^{+}}^{\alpha_{1}+\alpha_{2}+\alpha_{3} ; \varphi} g_{v}(b)\right|}{|\Delta| \Gamma\left(\alpha_{2}+\alpha_{3}+1\right) \Gamma\left(\alpha_{3}-\alpha_{4}+1\right)}
\end{aligned}
$$

by Lemma 4, and Proposition 1, we obtain

$$
\begin{aligned}
& |(\mathbb{T} u)(t)-(\mathbb{T} v)(t)| \\
\leq & \|u-v\|\left\{\frac{\delta K^{\alpha_{1}+\alpha_{2}+\alpha_{3}}}{\Gamma\left(\alpha_{1}+\alpha_{2}+\alpha_{3}+1\right)}+\frac{2 \mu K^{\alpha_{2}+2 \alpha_{3}}}{|\Delta| \Gamma\left(\alpha_{3}+1\right) \Gamma\left(\alpha_{2}+\alpha_{3}+1\right)}\right. \\
& +\frac{\mu K^{\alpha_{3}}}{\Gamma\left(\alpha_{3}+1\right)}+\frac{\mu K^{\alpha_{2}+2 \alpha_{3}}}{|\Delta| \Gamma\left(\alpha_{3}+1\right) \Gamma\left(\alpha_{3}+1\right) \Gamma\left(\alpha_{2}+\alpha_{3}-\alpha_{4}+1\right)} \\
& +\frac{\rho n K^{\alpha_{2}+\alpha_{3}}}{|\Delta| \Gamma\left(\alpha_{2}+\alpha_{3}+1\right) \Gamma\left(\alpha_{3}-\alpha_{4}+1\right)}+\frac{\rho n K^{\alpha_{2}+\alpha_{3}}}{|\Delta| \Gamma\left(\alpha_{3}+1\right) \Gamma\left(\alpha_{2}+\alpha_{3}-\alpha_{4}+1\right)} \\
& +\frac{\mu K^{\alpha_{2}+2 \alpha_{3}}}{|\Delta| \Gamma\left(\alpha_{2}+\alpha_{3}+1\right) \Gamma\left(\alpha_{3}+1\right) \Gamma\left(\alpha_{3}-\alpha_{4}+1\right)} \\
& +\frac{\delta K^{\alpha_{1}+2 \alpha_{2}+2 \alpha_{3}+\alpha_{4}}}{|\Delta| \Gamma\left(\alpha_{3}+1\right) \Gamma\left(\alpha_{2}+\alpha_{3}+1\right) \Gamma\left(\alpha_{1}+\alpha_{2}+\alpha_{3}+1\right)} \\
& +\frac{\delta K^{\alpha_{1}+2 \alpha_{2}+2 \alpha_{3}}}{|\Delta| \Gamma\left(\alpha_{3}+1\right) \Gamma\left(\alpha_{2}+\alpha_{3}-\alpha_{4}+1\right) \Gamma\left(\alpha_{1}+\alpha_{2}+\alpha_{3}+1\right)}
\end{aligned}
$$


$\leq \eta_{1}\|u-v\|$,

also

$$
\begin{aligned}
& \left|{ }^{c} D_{a^{+}}^{\alpha_{4} ; \varphi}(\mathbb{T} u)(t)-{ }^{c} D_{a^{+}}^{\alpha_{4} ; \varphi}(\mathbb{T} v)(t)\right| \\
\leq & \left|I_{a^{+}}^{\alpha_{1}+\alpha_{2}+\alpha_{3}-\alpha_{4} ; \varphi} g_{u}(t)-I_{a^{+}}^{\alpha_{1}+\alpha_{2}+\alpha_{3}-\alpha_{4} ; \varphi} g_{v}(t)\right|+\mu\left|I_{a^{+}}^{\alpha_{3}-\alpha_{4} ; \varphi} u(t)-I_{a^{+}}^{\alpha_{3}-\alpha_{4} ; \varphi} u(t)\right| \\
& +\frac{\left((\varphi(t)-\varphi(a))^{\alpha_{2}+\alpha_{3}-\alpha_{4}}+K^{\alpha_{2}}(\varphi(t)-\varphi(a))^{\alpha_{3}-\alpha_{4}}\right)}{|\Delta| \Gamma\left(\alpha_{3}-\alpha_{4}+1\right) \Gamma\left(\alpha_{2}+\alpha_{3}-\alpha_{4}+1\right)}|u(b)-v(b)| \\
& +\frac{\mu\left((\varphi(t)-\varphi(a))^{\alpha_{2}+\alpha_{3}-\alpha_{4}}+K^{\alpha_{2}}(\varphi(t)-\varphi(a))^{\alpha_{3}-\alpha_{4}}\right)\left|I_{a^{+}}^{\alpha_{3} ; \varphi} u(b)-I_{a^{+}}^{\alpha_{3} ; \varphi} u(b)\right|}{|\Delta| \Gamma\left(\alpha_{3}-\alpha_{4}+1\right) \Gamma\left(\alpha_{2}+\alpha_{3}-\alpha_{4}+1\right)} \\
& +\frac{\mu K^{\alpha_{2}+\alpha_{4}}(\varphi(t)-\varphi(a))^{\alpha_{3}-\alpha_{4}}\left|I_{a^{+}}^{\alpha_{3}-\alpha_{4} ; \varphi} u(b)-I_{a^{+}}^{\alpha_{3}-\alpha_{4} ; \varphi} u(b)\right|}{|\Delta| \Gamma\left(\alpha_{3}-\alpha_{4}+1\right) \Gamma\left(\alpha_{2}+\alpha_{3}+1\right)} \\
& +\frac{\mu K^{\alpha_{4}}(\varphi(t)-\varphi(a))^{\alpha_{2}+\alpha_{3}-\alpha_{4}}\left|I_{a^{+}}^{\alpha_{3}-\alpha_{4} ; \varphi} u(b)-I_{a^{+}}^{\alpha_{3}-\alpha_{4} ; \varphi} u(b)\right|}{|\Delta| \Gamma\left(\alpha_{3}+1\right) \Gamma\left(\alpha_{2}+\alpha_{3}-\alpha_{4}+1\right)} \\
& +\frac{\left(K^{\alpha_{2}}(\varphi(t)-\varphi(a))^{\alpha_{3}-\alpha_{4}}+(\varphi(t)-\varphi(a))^{\alpha_{2}+\alpha_{3}-\alpha_{4}}\right)\left|I_{a^{+}}^{\alpha_{1}+\alpha_{2}+\alpha_{3} ; \varphi} g_{u}(b)-I_{a^{+}}^{\alpha_{1}+\alpha_{2}+\alpha_{3} ; \varphi} g_{v}(b)\right|}{|\Delta| \Gamma\left(\alpha_{3}-\alpha_{4}+1\right) \Gamma\left(\alpha_{2}+\alpha_{3}-\alpha_{4}+1\right)} \\
& +\frac{K^{\alpha_{4}}(\varphi(t)-\varphi(a))^{\alpha_{2}+\alpha_{3}-\alpha_{4}}\left|I_{a^{+}}^{\alpha_{1}+\alpha_{2}+\alpha_{3}-\alpha_{4} ; \varphi} g_{u}(b)-I_{a^{+}}^{\alpha_{1}+\alpha_{2}+\alpha_{3}-\alpha_{4} ; \varphi} g_{v}(b)\right|}{|\Delta| \Gamma\left(\alpha_{3}+1\right) \Gamma\left(\alpha_{2}+\alpha_{3}-\alpha_{4}+1\right)} \\
& +\frac{K^{\alpha_{2}+\alpha_{4}}(\varphi(t)-\varphi(a))^{\alpha_{3}-\alpha_{4}}\left|I_{a^{+}}^{\alpha_{1}+\alpha_{2}+\alpha_{3}-\alpha_{4} ; \varphi} g_{u}(b)-I_{a^{+}}^{\alpha_{1}+\alpha_{2}+\alpha_{3}-\alpha_{4} ; \varphi} g_{v}(b)\right|}{|\Delta| \Gamma\left(\alpha_{3}-\alpha_{4}+1\right) \Gamma\left(\alpha_{2}+\alpha_{3}+1\right)}
\end{aligned}
$$

by Lemma 4, and Proposition 1, we obtain

$$
\begin{aligned}
& \left|{ }^{c} D_{a^{+}}^{\alpha_{4} ; \varphi}(\mathbb{T} u)(t)-{ }^{c} D_{a^{+}}^{\alpha_{4} ; \varphi}(\mathbb{T} v)(t)\right| \\
\leq & \|u-v\|\left\{\frac{\delta K^{\alpha_{1}+\alpha_{2}+\alpha_{3}-\alpha_{4}}}{\Gamma\left(\alpha_{1}+\alpha_{2}+\alpha_{3}-\alpha_{4}+1\right)}+\frac{\mu K^{\alpha_{3}-\alpha_{4}}}{\Gamma\left(\alpha_{3}-\alpha_{4}+1\right)}\right. \\
& +\frac{2 n \rho K^{\alpha_{2}+\alpha_{3}-\alpha_{4}}}{|\Delta| \Gamma\left(\alpha_{3}-\alpha_{4}+1\right) \Gamma\left(\alpha_{2}+\alpha_{3}-\alpha_{4}+1\right)} \\
& +\frac{3 \mu K^{\alpha_{2}+2 \alpha_{3}-\alpha_{4}}}{|\Delta| \Gamma\left(\alpha_{3}+1\right) \Gamma\left(\alpha_{3}-\alpha_{4}+1\right) \Gamma\left(\alpha_{2}+\alpha_{3}-\alpha_{4}+1\right)} \\
& +\frac{\mu K^{\alpha_{2}+2 \alpha_{3}-\alpha_{4}}}{|\Delta| \Gamma\left(\alpha_{3}-\alpha_{4}+1\right) \Gamma\left(\alpha_{3}-\alpha_{4}+1\right) \Gamma\left(\alpha_{2}+\alpha_{3}+1\right)} \\
& +\frac{2 \delta K^{\alpha_{1}+2 \alpha_{2}+2 \alpha_{3}-\alpha_{4}}}{|\Delta| \Gamma\left(\alpha_{3}-\alpha_{4}+1\right) \Gamma\left(\alpha_{2}+\alpha_{3}-\alpha_{4}+1\right) \Gamma\left(\alpha_{1}+\alpha_{2}+\alpha_{3}+1\right)} \\
& +\frac{\delta K^{\alpha_{1}+2 \alpha_{2}+2 \alpha_{3}-\alpha_{4}}}{|\Delta| \Gamma\left(\alpha_{3}+1\right) \Gamma\left(\alpha_{2}+\alpha_{3}-\alpha_{4}+1\right) \Gamma\left(\alpha_{1}+\alpha_{2}+\alpha_{3}-\alpha_{4}+1\right)} \\
& \left.+\frac{\delta K^{\alpha_{1}+2 \alpha_{2}+2 \alpha_{3}-\alpha_{4}}}{|\Delta| \Gamma\left(\alpha_{3}-\alpha_{4}+1\right) \Gamma\left(\alpha_{2}+\alpha_{3}+1\right) \Gamma\left(\alpha_{1}+\alpha_{2}+\alpha_{3}-\alpha_{4}+1\right)}\right\}
\end{aligned}
$$

Using (3.9) and (3.10), we obtain

$$
\|\mathbb{T} u-\mathbb{T} u\| \leq \eta\|u-v\|,
$$

where $\eta<1$. Hence the operator $\mathbb{T}$ is a contraction operator and the contraction mapping principle implies that the problem (1.1) has a unique solution. 
Example 1. Consider the following nonlinear Langevin equation of fractional orders

$$
\left\{\begin{array}{c}
{ }^{c} D_{a^{+}}^{\frac{1}{2} ; \varphi}\left({ }^{c} D_{a^{+}}^{\frac{1}{2} ; \varphi}\left({ }^{c} D_{a^{+}}^{\frac{1}{2} ; \varphi}+1\right)\right) u(t)=g\left(t, u(t),{ }^{c} D_{a^{+}}^{\frac{1}{3} ; \varphi} u(t)\right), t \in\left[\frac{1}{2}, 1\right] \\
\varphi(t)=t^{2} \\
g\left(t, u(t),{ }^{c} D_{a^{+}}^{\frac{1}{3} ; \varphi} u(t)\right)=t+\left(t-\frac{1}{8}\right)^{2}(u(t))^{\delta_{1}}+\frac{t}{6}\left({ }^{c} D_{a^{+}}^{\frac{1}{3} ; \varphi} u(t)\right) \\
0<\delta_{1}, \delta_{2}<1
\end{array}\right.
$$

Observe that the function $g$ is continuous, also

$$
\left|g\left(t, u(t),{ }^{c} D_{a^{+}}^{\frac{1}{3} ; \varphi} u(t)\right)\right| \leq 1+\frac{49}{64}|u(t)|^{\delta_{1}}+\frac{1}{6}^{c} D_{a^{+}}^{\frac{1}{3} ; \varphi}|u(t)|^{\delta_{2}}
$$

Thus, the assumptions $\left(\mathbb{H}_{1}\right)$ and $\left(\mathbb{H}_{2}\right)$ are satisfied and Theorem 1 implies that the problem (3.11) has a solution.

\section{REFERENCES}

[1] A. Aghajani, E. Pourhadi, J. Trujillo, Application of measure of noncompactness to a Cauchy problem for fractional differential equations in Banach spaces, Fract. Calc. Appl. Anal. 16 (2013) 962-977. https: //doi.org/10.2478/s13540-013-0059-y.

[2] O. Agrawal, Some generalized fractional calculus operators and their applications in integral equations, Fract. Calc. Appl. Anal. 15 (2012) 700-711. https://doi.org/10.2478/s13540-012-0047-7.

[3] B. Ahmad,M.M.Matar and O.M. EL-Salmy, Existence of solutions and ulam stability for Caputo type sequential fractional differential equations of order $\alpha \in(2,3)$, Int. J. Anal. Appl. 15 (2017) 86-101.

[4] R. Almeida, A Caputo fractional derivative of a function with respect to another function, Commun. Nonlinear Sci. Numer. Simul. 44 (2017) 460-481. https://doi.org/10.1016/j .cnsns. 2016.09.006.

[5] R. Almeida, A.B. Malinowska, M.T.T. Monteiro, Fractional differential equations with a Caputo derivative with respect to a Kernel function and their applications, Math. Meth. Appl. Sci. 41 (2018) 336-352. https://doi.org/10.1002/mma.4617.

[6] A. Benzidane, Z. Dahmani, A class of nonlinear singular differential equations, J. Interdiscip. Math. 22 (2019) 991-1007. https://doi.org/10.1080/09720502.2019.1698805.

[7] M. Bezziou, Z. Dahmani, A. Ndiaye, Langevin differential equation of fractional order in non compactness Banach space, J. Interdiscip. Math. 23 (2020) 857-876. https://doi.org/10.1080/09720502 . 2020. 1730515.

[8] Z. Dahmani, L. Marouf, Numerical study of differential equation governing speech gestures with caputo derivative, J. Interdiscip. Math. 16 (2013) 287-296. https://doi.org/10.1080/09720502 .2013.821587.

[9] K. Diethelm, The Analysis of Fractional Differential Equations: An Application-Oriented Exposition Using Differential Operators of Caputo Type, Lecture Notes in Mathematics, Springer-Verlag, Berlin, 2010.

[10] H. Fazli, J.J. Nieto, Fractional Langevin equation with anti-periodic boundary conditions, Chaos Solitons Fractals. 114 (2018) 332-337. https://doi.org/10.1016/j.chaos.2018.07.009.

[11] R. Herrmann, Fractional Calculus for Physicist, World Scientific, Singapore, 2014.

[12] M.D. Kassim, N.-E. Tatar, Stability of logarithmic type for a Hadamard fractional differential problem, J. Pseudo-Differ. Oper. Appl. 11 (2020) 447-466. https://doi.org/10.1007/s11868-019-00285-3.

[13] A.A. Kilbas, H.M. Srivastava, J.J. Trujillo, Theory and applications of fractional differential equations, North-Holland Mathematics Studies, vol. 204. Elsevier Science, Amsterdam, 2006

[14] S. Kosari, M. Yadollahzadeh, Z. Shao, Y. Rao, On a generalization of fractional Langevin equation, ArXiv:2004.03542 [Math]. (2020). http://arxiv.org/abs/2004.03542.

[15] T.J. Osler, The fractional derivative of a composite function, SIAM J. Math. Anal. 1 (1970) 288-293. https://doi.org/10.1137/0501026.

[16] I. Podlubny, Fractional Differential Equations, Academic Press, San Diego, 1999. 
[17] H. Qin, X. Zuo, J. Liu, Existence and controllability results for fractional impulsive integrodifferential systems in Banach spaces, Abstr. Appl. Anal. 2013 (2013) 295837. https://doi.org/10.1155/2013/295837.

[18] S.G. Samko, A.A. Kilbas, O.I. Mariche, Fractional integrals and derivatives, translated from the 1987 Russian original. Gordon and Breach, Yverdon, 1993.

[19] [1]A. Seemab, J. Alzabut, M. ur Rehman, Y. Adjabi, M.S. Abdo, Langevin equation with nonlocal boundary conditions involving a $\psi$-Caputo fractional operator, ArXiv:2006.00391 [Math]. (2020). http://arxiv. org/abs/2006.00391. 